\title{
Feuilleton.
}

Karl Krall, Denkende Tiere. Beiträge zur Tierseelenkunde auf Grund eigener Versuche. Leipzig, Engelmann, 1912. Besprochen von H. E. Zi egler (Stuttgart).

Der Ieser wird vielleicht dieses Buch mit Mißtrauen in dic Hand nehmen, aber je: inehr er sich himein vertieft, $4 \mathrm{~m}$ so mehr wird er dem Verfasser Vertranen sehenken und von den stannenswerten Mitteilungen iiberrascht sein. In der Tat ist hier der Bewcis geliefert, cla B die Pferde einen viel höheren Verstand haben, als nan bisher anzmehmen geneigt war, und daß sic wirkliehren Denkens: fähig sind, das sich nicht allein in cincr fast unglanblichen Fähigkeit des Rechmcns, sonclern anch im Buchstabieren von Worten und in verselicelenen anderen morkwürdigen Verstandesäußermugen zeigt. Un diese bis jetzt mbekanuten lähigkeitcu zn entdeeken, war es ror allem nötig, clem Tiere durch Unterricht die Mögliehkeit \# verschaffen, seinen Gedanken Ansdruck \%u geben. Die Zahlen werden durch Klopfen mit den VorderfïBen anf einen ., Klopfbrett" angegeben, wobei der reehte Fuß die Eincr. der linke dic Zelmer angibt. Die Buehstaben werden ebenfalls duch Zahlen bercichnet, allerdings nicht naeh der Nitellung in Alphabet, sondern nach einer Tabelle, cbenso wie man an ciner Schreibmaschine jeden Buchstaben durch aie Kolome in der Klaviatur und dmeh die Ordnungswahl in der Kolome bestimmen kömute; jeder Buchstahe wird also durch \%wei Zahlen angegeben.

Diese Methoden wurden schon von Herrn v. Osten bei clem, ,klugen Hans" angewandt mol sind mu von Herm $K$ rall noch vorbessert worden. Schon jenes vor einigen Jahren berühnt gewordene Pferd gab erstaunliche Beweise seiner Fäligkeiten, aber scin Rulm sehwand rasch clahin, als die Psychologen die Belianptung anfstcoltten, daß das Tier ïberhaupt keine nenuenswerte Denkfähigkeit besit\%e, sondern jedesmal das Resultat der Aufgabe nach Art der Gedankenleser ans mwillkürlichen Bewegungen seines Isehmneisters crkenne.') Der angebliche Beweis für diese Ansicht lag darin, daß dic Erfolge ausblieben, als man dem Pferde Sehenleder angezogen hatte, sodaß es denjenigen, der die Anfgabe stellte, nicht mehr sehen konnte.

Aber Kiall, der Verfasser des vorliegenden Buclies. hatte sich durch sorgfältige Beobachtung ïberzengt, daß das Pferd doeh cine

1) Auf Veranlassung von Geheimrat Prof. Dr. Stumpf (Berlin) hat Dr. P'fungst ein ansführliches Gutachten über das Pferd verfaßt (Pfungst, Das Pferd des Herrn v. Osten, 1907). Es muß aber Bedenken gegen dieses Gntachten erwcoken, daß Pfungst den klugen Hans absichtlich dazu angelcitet hat, auf Zeichen $\mathrm{n}$ antworten; ich halte dieses Verfahren für irreführend. 
erstannliche eigene Denkfähigkeit besaß. Fr erkannte, daß die Sichenklappen für das Pferd einc nngewolnte Störung bildeten, sodaß es da. durch von seiner Verstandesarbeit abgelenkt wurde. Er gewöhnte das Ticr allmählich an die Sicheuklappon und erziclte nun wieder dieselben gutcon Resultate wie frühcr, anch wenn die Person, welele dic Aufgabe stcllte und das Ergebuis beobaelitcte, von dem I'ferd nicht gesehen werden konnte. Damit war erwiescn, da $B$ die einfache Erklärung jener Psychologen nicht zutreffend war.

K rall unterriehtete nun \%wei andere Pferde, junge Araber-Hengste, welehe so rasehe Fortsehritte machten, da $\beta$ der Lelırmeister selbst davon iiberracelit war. Sehon an dritten Tage der Unterweisung hoben sic auf Befehl den rechten oder den linken FuB, wic man es verlangte. Selion nach 14. Tagen führten sie Additionen, Subtraktionen und Multiplikationen nit klcincen Zahlen (bis zur Zahl 10) ganz richtig ans. Die Fortscliritte in Rechnen wurden bald erstaunlich. Schon im ersten Jahr relıneten sie größBere Divisionen richtig aus (\%. B. 8924671 : $6=$ 1487445, Rest 1). Auch kannten sie schon eine An $\%$ ahl Quadratzahlen, sodaß sie von xwcistelligen und dreistelligen Zahlen dis Quadratwurzelı angeben konnten. Ferner lernten sic: im ersten Jahr sehon das Buchstabieren in der oben angedenteten Weise. Dabei gesehah das Merkwïrdige, daß die Pferde nach dem Klang der Wörter buchstabierten und dadurch zu einer gan\% cigenartigen Sichreibweise kamen; darin liegt eben ciner der Bewcise fiir das selbständige Denken der Tiere; denı diese sichreibweise kommt gan\% uncrwartet und fällt durchans nicht immer gleichartig aus; $\%$. B. sehreiben sie das Wort Pferd als pfärt, färd, ferd, pärd oder fräd odcr auch noch auf anderc Arten. Sic lassen meistens die Vokale ans, welche schion in den Namen der Buchstaben cuthalten sind, $\%$. B. hfr gbn (statt Hafer geben) oder ig $\mathrm{m}$ hlon (statt ich Mölıren haben).

Gcrade dicse Fehlerhaftigkcit und Mannigfaltigkcit zeigt, da B kcin (infaches Dressurresultat vorliegt; denn das Wesen der Dresinl ist dic stets gleichniäBige Wiederholung; das Resultat derselben verlänft also stets genau in der vorherzusagenden Weisc, wälırend das cigene Denken oft x.l einem nnerwarteten Ergelunis führt.

In der Tat waren die Antworten der Pferde in unzähligen Fällen fiir alle Cimstehenden durchaus inberraselend. Dadureh ist bewiesen, dals irgendwelche Zcichengebung oder irgendeine Suggestion nicht in Betracht komnt.') In nenerer Zcit sind dic Pferde von vielen Besuchen geprïft worden. und manehe derselben hatten Rechenanfgaben mitgebracht. deren Lösmng keincm der Anwesenden bekannt war und wegen der Sichwicrigkeit der Aufgabe auch nicht im Kopfe ansgerechnet werden konnte. Trotzdem wurde das Resultat von einem der P'f(rde richtig angegebe:n. Da bleibt keinc andere: Möglichkeit: man muß das cigene: De'nken der Tiere ancrkennen. ${ }^{2}$ )

Das Merkwürdigste ist, daß dic Ticre sich des erlernten Buchstabiercns bedienten, um cigene (iedanken anszudriicken. Z. B. hatte cin Besneher einen l'ferdekopf mit offenem Mnnde an die Tafel gezeielmet, worauf eines der Pferde von sich aus die Worte buelstabicrte ang b) ghin ferd davel (d. lo, aueh Brot gelon dem Pferd an der Tafel).

Herrn v. Osten muß das Verdienst zncrkannt werden, encrst ge\%eigt \%n haben, daß dic Pferde bei passender Anlcitung des Rechnens und des Buchstabicrens fälig sind. Nachdem scine Anschanungen über die Leistmngen des Pferdes cine scheinbare Widerlegung erfahren haben, kommen sie nun nach seinem Tode wieder \%on illrem Recht. Herr Krall hat mit bewundernswerter Ausdaner und großcon Gesehick dic

1) Einen

solchen Fall beriehtet anch ncuerdings Prof. Kränıer (Hohenheim) in der Bergisch-1Iärkischen Zcitung (10. April 1912)

„An der Tafel stehen Ziffern in verschiedenen Farben; wuf den Befehl, die zwei rechtsstehenden Zahlen zusammenzuzählen, gibt das Pferd eine unerwartete Antwort. Falseh heißt es; doch das Pferd bleibt bei seiner Mcinung. Da sche ich plötzlich, da B etwas weiter rechts anf cinem Fernsprecher in glcicher Iröllc: noch ein Täfelchen mit der Zahl 5 anf Zufall stehengeblieben ist. Dicse hat der Hengst mitgezählt und bei diescr Auffassung der Frage war er subjektiv völlig im Recht

U'm Suggestion oder Dressur konnte es sich nieht handeln, denn wir alle hatten iibereinstimmend eine andere Lösung erwartet."

Ein reeht interessantes Beispiel erzälılt der Arzt Dr. Hermann Dekker in „,Kosmos“. Den Namen buchstabierte das Pferd mit den drei Buchstaben dgr. Das l'ferd wurde von seinem Herrn aufgefordert, zil sagen, was (s an dem Gaste bcinerke. Man erwartete das Wort Schnurrbart. Das Pferd antwortete aber "Sehmeren imbnn". Es zeigte sich, daß es sagen wollte "Schmerzen im Bein". Das andere Pferd hatte nämlich Schmerzen im Beill. Aus einigen weiteren Fragen ging mit Sicherheit hervor, daß das 'Tier bei dem Doktor an cinen Tieraryt und an das Leiden des anderen Pferdes gedacht hatte. Es fügte sogar selbständig die Worte hinzu: midman albrd banntwasr, d. h. der Tierarzt Mittmann hat den Pferdewärter Albert beaıltragt, ein Band mit Wasser aufzulegen

2) Gan\% unerklärt ist zurzeit dic Fähigkeit des Wurzelausziehens. Neuerdings wird berichtet, daß das eine der Pferde auch vierte und fünfte Wurzeln richtig angegeben hat. Die Methode des Wurzelauszichens ist den Tieren überhaupt nieht gelehrt worden. Da nach den Versuehsbedingungen eine Zeiehengebung völlig ausgeschlossen ist, bleibt diese Fähigkeit zurzeit noch rätselhaft.
Versinche fortgesetzt and so staunenswerte Ergebnisse criclt. Sein Werk bedentct einen ungeahnten Fortschritt anf dem (iebiet der Tierpsycholugie, für welchen ihm die Wisicnsclaaft dankbar sein muß.

\section{Nachwort der Redaktion.}

Die Anerkennung, die Prof. Ziegler, der selır verdiente friihere Jenenser, jet tet in Stuttgart lehrende Prof. der Zoologic, den Untersnchungen von $v$. Osten nnd Krall ïber ihre denkenden Pferde zollt, ist sicherlich wohl $\%$ beachten. Sein Urtcil stelıt im Einklang mit andcren Beobachtern, von dencen wir nur Edinger anführen wollen, 11m \%u zeigen, daß es sich anch konst nun Männer vou hoher Autorität handelt. Anderseits ist darauf lin\%nweisen, daß dic schon an nnd für sich unseren bisherigen Ansehanungen üler das Tiergehirn stark wider. sprechenden, höchst nnwahrseheinlich klingenden Angaben Kralls von Psvehologen (wie Stumpf und seinen Sehiilern) und Zoologen (\%. B. Prof. Dexler in Prag; vgl. No. 25, S. 1201) aufs sehärfstc bekämpft werden. Freilich sind die Erklärnngen, dic diese Forscher für dic nnzweifelhaft feststehenden, verbliiffenden Prüfungsresultate der Pforde geben, nicht befriedigend: nit cincm sipiel des ,Zu. falls", Er\%eugnissen der ,Dressur", ,unbewnßten Becinflussungen“ durch den Fragestchler lassen sich die nutcr den verschiedensten Versuchsbedingmngen sieh wiederhokenden Tatsachen nicht genügend deuten. Wcitere stndien iiber dicse rätsellhaften Erscheimmgen werden wohl Lieht in das: Dunkel bringen. 